\title{
Risks of potential drug-drug interactions in COVID-19 patients treated with corticosteroids: a single-center experience
}

\author{
D. Cattaneo $0^{1,2} \cdot$ L. Pasina $^{3} \cdot$ F. Conti ${ }^{4} \cdot$ A. Giacomelli ${ }^{4} \cdot$ L. Oreni $^{4} \cdot$ L. Pezzati ${ }^{4} \cdot$ C. Bonazzetti ${ }^{4} \cdot$ M. Piscaglia $^{4}$. \\ G. Carrozzo ${ }^{4} \cdot$ S. Antinori ${ }^{4} \cdot$ C. Gervasoni ${ }^{1,4}$
}

Received: 16 April 2021 / Accepted: 22 May 2021 / Published online: 30 May 2021

(c) Italian Society of Endocrinology (SIE) 2021

\section{Introduction}

A high rate of polypharmacy is expected in COVID-19 patients as the result of the treatments of existing agingrelated chronic disease conditions and the medications tested for SARS-CoV-2 infection [1]. Furthermore, patients hospitalized for COVID-19 may receive other drugs for the treatment of specific symptoms, further aggravating their overall pharmacological burden and the risk for potential drug-drug interactions (DDIs). Taken together, these evidences put COVID-19 patients at extremely high risk for experiencing potentially severe DDIs during hospital stay. We have recently demonstrated that, during the first pandemic wave, more than $60 \%$ of patients with COVID-19 were exposed to at least one potential DDI, and the proportion of patients experiencing a potentially severe DDI increased significantly from $20 \%$ at admission to $80 \%$ during hospitalization [2].

During the second COVID-19 outbreak, occurring in Italy in the last months of 2020, corticosteroids were widely used in the management of patients affected by severe acute respiratory distress syndrome caused by SARS-CoV-2 infection $[3,4]$. Beyond the potential risk of adrenal insufficiency, the use of corticosteroids in the context of hospitalized COVID19 patients has been a matter of active debate mainly for the

C. Gervasoni

cristina.gervasoni@asst-fbf-sacco.it

1 Gestione Ambulatoriale Politerapie (GAP) Outpatient Clinic, ASST Fatebenefratelli-Sacco University Hospital, Milan, Italy

2 Unit of Clinical Pharmacology, ASST Fatebenefratelli-Sacco University Hospital, Milan, Italy

3 Istituto Di Ricerche Farmacologiche Mario Negri IRCCS, Milan, Italy

4 Department of Infectious Diseases, ASST

Fatebenefratelli-Sacco University Hospital, Via GB Grassi

74, 20157 Milano, Italy risk of DDIs with concomitant treatments [3, 4]. In fact, corticosteroids are inducers of liver enzymes involved in the metabolism of several drugs [4]. In this report, we aim to assess the risk of corticosteroid-related potential DDIs in COVID-19 patients hospitalized at the ASST Fatebenefratelli-Sacco University Hospital during the second pandemic wave.

\section{Materials and methods}

We searched the database of the Department of Infectious Diseases of Luigi Sacco Hospital (Milan, Italy) for patients with a proven diagnosis of SARS-CoV-2 infection (a throat swab positive for viral nucleic acid) hospitalized between September 30, 2020 and December 31, 2020, treated with at least two drugs and with available information concerning pharmacological treatments during hospitalization. The risk of potential DDIs was assessed using INTERcheck, a Computerized Prescription Support System which classifies them according to their clinical relevance as follows: class D (contraindicated: drug combinations that should be avoided); class $\mathrm{C}$ (major: drug combinations requiring close monitoring for potentially serious clinical consequences, such as severe adverse effects or lack of clinical efficacy); class B (moderate: drug combinations requiring dose adjustment and/or drug concentration monitoring); class A (minor: drug combinations with no known clinical relevance) [2]. Potentially severe DDIs were defined as the sum of class D and class $\mathrm{C}$.

The study was approved by our hospital's Ethics Committee (Comitato Etico Interaziendale Area (1). The frequency distribution data are expressed as absolute numbers and percentages, and all of the other measures as mean values \pm standard deviation. 


\section{Results}

Six-hundred-and-twenty-eight COVID-19 patients fulfilling the inclusion criteria were identified. Male gender predominated (64\%) and the mean age was $67 \pm 16$ years. During hospitalization, they received a mean of $7.0 \pm 4.1$ drugs. Overall, $72 \%$ of the enrolled patients were exposed to at least one potential DDI, $48 \%$ of which were classified as potentially severe.

Seventy-five percent of the patients $(n=471)$ were treated with a corticosteroid, mainly dexamethasone $(87 \%)$, prednisone (4\%), beclomethasone (3\%) or methylprednisolone (2\%). Potential DDIs with concomitant therapies $(n=781)$ were found in 345 out of the 471 patients (73\%) on corticosteroids. No class D DDIs were recorded. Conversely, 25 and 756 class C and class B potential DDIs involving corticosteroids were, respectively, identified. As shown in Table 1, class C DDIs were mainly driven by caspofungin $(60 \%)$ and voriconazole (24\%), increasing the risk of reduced antifungal exposure and drug efficacy according to available literature $[5,6]$.

The interacting agents involved in class B potential DDIs were more largely distributed (Table 2), eventually resulting in reduced exposure and efficacy of antihypertensive agents (35\%), hypokalemia (18\%), bleeding $(17 \%)$ and impaired activity of the antiviral remdesivir (13\%) or hypoglycemic agents (11\%). Concomitant administration of corticosteroids and the antibiotic drug class of fluoroquinolones resulted in increased risk of tendon rupture in $2 \%$ of patients. Detailed information on the DDIs involving corticosteroids (mechanisms, level of evidences, etc.) can be found in the INTERcheck website after free registration (https://intercheckweb. marionegri.it/).

\section{Discussion}

This study first confirms that, also during the second SARS-CoV-2 outbreak, hospitalized COVID-19 patients were potentially exposed to clinically relevant DDIs, with severe DDIs being identified in nearly $50 \%$ of patients [2]. Moreover, we extended previous findings by documenting
Table 2 Potential class B drug-drug interactions $(n=756)$ in hospitalized COVID-19 patients treated with corticosteroids $(n=471)$

\begin{tabular}{ll}
\hline Potential adverse event/interacting agents & $N(\%)$ \\
\hline Antagonism of the action of antihypertensive drugs & $267(35 \%)$ \\
Beta-blockers & 110 \\
ACE inhibitors & 82 \\
Angiotensin II receptor antagonists & 50 \\
Alpha 1 blockers & 15 \\
Calcium channel blockers & 7 \\
Diuretics & 3 \\
Hypokalemia (lethargy, asthenia, arrhythmias) & $139(18 \%)$ \\
Diuretics & 105 \\
Beta agonists & 34 \\
Bleeding & $130(17 \%)$ \\
Acetylsalicylic acid & 116 \\
Vitamin K inhibitors & 14 \\
Reduced exposure and efficacy of remdesivir & $97(13 \%)$ \\
Reduced exposure and efficacy of hypoglycemic agents & $81(11 \%)$ \\
Metformin & 65 \\
Glinides & 9 \\
Incretin mimetics & 7 \\
Increased risk of tendon rupture & $15(2 \%)$ \\
Fluoroquinolones & 15 \\
Others & $27(4 \%)$ \\
Quetiapine & 16 \\
Antiepileptic drugs & 6 \\
Others & 5 \\
\hline
\end{tabular}

that corticosteroids, prescribed in the majority of patients during the second pandemic wave, had only a marginal effect on the risk of DDIs. In fact, the use of these drugs did not result in contraindicated drug combinations, with major DDIs being identified only in 5\% of treated patients. Considering that the inductive effect of corticosteroids on cytochromial enzymes is time- and dose-dependent, the clinical impact of these DDIs might be limited in COVID19 patients treated with $6 \mathrm{mg}$ of dexamethasone for 10 days in most cases. This may be a reassuring message for both patients and attending physicians, confirming the safe use of corticosteroids in the setting of COVID-19, at least for the risk of major DDIs. Nevertheless, we believe that it is
Table 1 Potential class $\mathrm{C}$ drugdrug interactions $(n=25)$ in hospitalized COVID-19 patients treated with corticosteroids $(n=471)$

\begin{tabular}{llc}
\hline Potential adverse event & Interacting agent & $N(\%)$ \\
\hline Reduction of the exposure and efficacy of caspofungin & Caspofungin & $15(60)$ \\
Reduction of the exposure and efficacy of voriconazole & Voriconazole & $6(24)$ \\
Increased risk of infections & Adalimumab & $1(4)$ \\
Increased risk of gastrointestinal adverse effects & Deferasirox & $1(4)$ \\
Reduction of exposure to efavirenz and/or corticosteroids & Efavirenz & $1(4)$ \\
Increased risk of gastrointestinal adverse effects & Ketorolac & $1(4)$ \\
\hline
\end{tabular}


equally important to acknowledge that the addition of corticosteroids to background therapies resulted in a dramatic increase in the number of DDIs classified as moderate (drug combinations requiring dose adjustments and/or drug concentrations monitoring).

The need for physicians to remain vigilant for the potential DDIs involving corticosteroids is reinforced by recent evidences showing that the disposition of some drugs is significantly altered by the presence of SARS-CoV-2-related pro-inflammatory state which reduced the activity of metabolic enzymes [7, 8]. Accordingly, it cannot be excluded that DDIs predicted to be moderate in SARS-CoV-2-uninfected patients might eventually become clinically relevant in some phases of the COVID-19 disease. Indeed, during the active phase of SARS-CoV-2 infection, pro-inflammatory cytokines are likely to downregulate the activity of liver enzymes, eventually counterbalancing the well-known inductive effect of corticosteroids on drug metabolism [9]. However, when the inflammatory phase ends, corticosteroid-related DDIs may be revealed, raising potential clinical challenges. These events should be carefully considered and properly handled by physicians involved in the management of COVID-19, from admission of patients to the hospital to their discharge.

Authors' contributions Research design and manuscript first draft: DC, CG. Provision of study materials or patients: FC, AG, LP, CB, MP, GC, SA, CG. Data analysis: DC, LP, LO. Final manuscript approval: All authors.

Funding No sources of funding were used to conduct this study or prepare the manuscript.

\section{Declarations}

Conflict of interest We declare no competing interest for the present study. CG has received personal fees from MSD, ViiV, Gilead and Janseen Cilag unrelated to this study. DC has received personal fees from MSD, ViiV, and Janseen Cilag unrelated to this study. All of the other authors declare that they have no potential conflict of interest.

Ethical approval The study was approved by our hospital's Ethics Committee (Comitato Etico Interaziendale Area 1).
Consent to participate All of the patients included in the study signed an informed consent form.

\section{References}

1. Awortwe A, Cascorbi I (2020) Meta-analysis on outcome-worsening comorbidities of COVID-19 and related potential drug-drug interactions. Pharmacol Res. https://doi.org/10.1016/j.phrs.2020. 105250

2. Cattaneo D, Pasina L, Maggioni AP, Giacomelli A, Oreni L, Covizzi A, Bradanini L, Schiuma M, Antinori S, Ridolfo A, Gervasoni C (2020) Drug-Drug interactions and prescription appropriateness in patients with COVID-19: a retrospective analysis from a reference hospital in Northern Italy. Drugs Aging 37(12):925-933

3. Berton AM, Prencipe N, Giordano R, Ghigo E, Grottoli S (2021) Systemic steroids in patients with COVID-19: pros and contras, an endocrinological point of view. J Endocrinol Invest 44(4):873-875

4. Ferraù $F$, Ceccato F, Cannavò S, Scaroni C (2021) What we have to know about corticosteroids use during Sars-Cov-2 infection. J Endocrinol Invest 44(4):693-701

5. Wallace KL, Filipek RL, La Hoz RM, Williamson JC (2016) Subtherapeutic voriconazole concentrations associated with concomitant dexamethasone: case report and review of the literature. J Clin Pharm Ther 41(4):441-443

6. Li CC, Sun P, Dong Y, Bi S, Desai R, Dockendorf MF, Kartsonis NA, Ngai AL, Bradshaw S, Stone JA (2011) Population pharmacokinetics and pharmacodynamics of caspofungin in pediatric patients. Antimicrob Agents Chemother 55(5):2098-2105

7. Baldelli S, Corbellino M, Clementi E, Cattaneo D, Gervasoni C (2020) Lopinavir/ritonavir in COVID-19 patients: maybe yes, but at what dose? J Antimicrob Chemother 75(9):2704-2706

8. Cojutti PG, Londero A, Della Siega P, Givone F, Fabris M, Biasizzo J, Tascini C, Pea F (2020) Comparative population pharmacokinetics of Darunavir in SARS-CoV-2 patients vs HIV patients: the role of interleukin-6. Clin Pharmacokinet 59(10):1251-1260

9. Deb S, Arrighi S (2021) Potential effects of COVID-19 on cytochrome P450-mediated drug metabolism and disposition in infected patients. Eur J Drug Metab Pharmacokinet 46(2):185-203

Publisher's Note Springer Nature remains neutral with regard to jurisdictional claims in published maps and institutional affiliations. 